TITLE:

Above-threshold ionization of $\mathrm{Mg}$ by linearly and circularly polarized laser fields: Origin of the subpeaks in the photoelectron energy spectra

AUTHOR(S):

Nakajima, T; Buica, G

CITATION:

Nakajima, T ... [et al]. Above-threshold ionization of Mg by linearly and circularly polarized laser fields: Origin of the subpeaks in the photoelectron energy spectra. PHYSICAL REVIEW A 2006, 74(2): 023411.

ISSUE DATE:

2006-08

URL:

http://hdl.handle.net/2433/50420

RIGHT:

Copyright 2006 American Physical Society 


\title{
Above-threshold ionization of Mg by linearly and circularly polarized laser fields: Origin of the subpeaks in the photoelectron energy spectra
}

\author{
Takashi Nakajima* and Gabriela Buica ${ }^{\dagger}$ \\ Institute of Advanced Energy, Kyoto University, Gokasho, Uji, Kyoto 611-0011, Japan \\ (Received 9 June 2006; published 25 August 2006)
}

\begin{abstract}
We theoretically investigate above-threshold ionization of $\mathrm{Mg}$ by linearly and circularly polarized fs laser pulses. We find that the above-threshold ionization peaks are accompanied by small subpeaks for both linearly and circularly polarized pulses. We interpret the physical origin of the subpeaks as above-threshold ionization from the low-lying bound states which are far off-resonantly excited by the spectral wing of the pulse. This interpretation is confirmed by our comparative numerical studies. Furthermore, we provide a clear explanation of why this kind of subpeak in the photoelectron energy spectra has not been reported for smaller photon energies with $\mathrm{Mg}$ and other commonly used atoms such as $\mathrm{H}$ and rare gas atoms.
\end{abstract}

DOI: 10.1103/PhysRevA.74.023411

PACS number(s): $32.80 . \mathrm{Rm}, 42.50 . \mathrm{Hz}$

\section{INTRODUCTION}

Above-threshold ionization (ATI), a well-studied process by now $[1,2]$, is a process in which atoms exposed to an intense laser field absorb more than the minimum number of photons required for ionization. An ATI spectrum consists of a series of peaks, equally separated by the photon energy, in a photoelectron energy spectrum (PES). It has been found that the ATI spectra may present interesting features such as peak suppression [3], dynamic resonances [4], and substructures [5], etc., depending on the atoms or molecules employed and the laser parameters such as photon energy, peak intensity, pulse duration, and laser polarization, etc. Indeed, there are quite a few experimental studies $[3,6-8]$ in the context of ATI using both linearly and circularly polarized lasers, since such a comparison is very useful to understand the ionization dynamics. In contrast, most of the theoretical studies $[9,10]$ which deal with a circularly as well as linearly polarized field assume a strong laser field, which somehow simplifies the argument because ionization takes place essentially through the tunneling process $[11,12]$, or in the strong field limit, even the existence of the core may be neglected which leads to the so-called Volkov solution [13]. If the intensity is lower (typically $<10^{13} \mathrm{~W} / \mathrm{cm}^{2}$ ), the above theoretical treatments are not obviously valid. This is the so-called multiphoton ionization regime and one may have to solve the time-dependent Schrödinger equation (TDSE) to predict the behavior of the system. Note that the method based on TDSE is applicable from the low to high intensity range, since it relies on the direct integration of TDSE. Of course, for even lower intensity, the time-independent perturbation theory may be valid for the linearly as well as circularly polarized laser field [14-17]. It is not a priori obvious, however, whether such theoretical treatments are capable of predicting all the detailed features one can find from the solution of TDSE. In this context, we note that, except for a few works [18], most of the theoretical work reported in the literature,

\footnotetext{
*Email address: t-nakajima@iae.kyoto-u.ac.jp

†Permanent address: Institute for Space Sciences, P.O. Box MG-23, Ro 77125, Bucharest-Măgurele, Romania.
}

which utilizes computer codes to solve the TDSE as a machinery, assume a linearly polarized laser field.

The purpose of this paper is to theoretically investigate the ionization dynamics of $\mathrm{Mg}$ for the linearly polarized (LP) as well as circularly polarized (CP) laser field by solving the TDSE. Although our theoretical treatment allows us to go into the intensity range of so-called tunneling ionization regime, we restrict ourselves within the multiphoton ionization regime to avoid unnecessary complications. The photon energies we assume in this paper correspond to the second and third harmonics of a Ti:sapphire laser. After performing numerical calculations, we find that the PES exhibits not only the ordinary ATI peaks but also subpeaks between them. A similar trend is found for both second and third harmonic pulses. To clarify the origin of the subpeaks in the PES, extensive discussions are made and we successfully identify that the subpeaks originate from the low-lying bound states which are far off-resonantly excited by the spectral wing of the pulse. In other words, main (ordinary) peaks are the ATI signals from the ground state, while subpeaks are the ATI signals from the low-lying bound state(s). Based on this interpretation, it is clear that, depending on the photon energy, different bound states contribute to the subpeaks. It should be noted that the theoretical method we employ, i.e., solving the TDSE on the atomic basis states, enables us to easily identify the origin of the subpeaks.

This paper is organized as follows. In Sec. II we present a theoretical model: The time-dependent Schrödinger equation, which describes the time-dependent interaction dynamics, is solved on the discretized atomic basis states with two active valence electrons of $\mathrm{Mg}$. Atomic units are used in this section. In Secs. III and IV we present representative numerical results for the linearly and circularly polarized second and third harmonics of a Ti:sapphire laser. Comparative numerical results are presented to confirm our interpretation regarding the origin of the subpeaks in the PES. Finally, concluding remarks are given in Sec. V.

\section{TIME-DEPENDENT SCHRÖDINGER EQUATION}

In order to study the interaction of $\mathrm{Mg}$, a two-valenceelectron system, with a fs laser pulse, we solve the TDSE on 
the discretized atomic basis constructed in a spherical box. As we will show in the following sections, time propagation of the total wave function on the atomic basis [19-22] enables us to pinpoint the physical origin of the dynamics of interest, which is actually the main advantage of this method compared to other methods which propagate the numerical wave function itself. Now the TDSE for the two-electron system reads

$$
i \frac{d}{d t} \Psi\left(\mathbf{r}_{1}, \mathbf{r}_{2} ; t\right)=\left[H_{a}\left(\mathbf{r}_{1}, \mathbf{r}_{2}\right)+D(t)\right] \Psi\left(\mathbf{r}_{1}, \mathbf{r}_{2} ; t\right),
$$

where $\Psi\left(\mathbf{r}_{1}, \mathbf{r}_{2} ; t\right)$ represents the total wave function at time $t$ for the two-valence electrons at positions $\mathbf{r}_{1}$ and $\mathbf{r}_{2}$. The field-free Hamiltonian $H_{a}$ can be expressed as

$$
H_{a}\left(\mathbf{r}_{1}, \mathbf{r}_{2}\right)=\sum_{i=1}^{2}\left[-\frac{1}{2} \nabla_{i}^{2}+V_{e f f}\left(\mathbf{r}_{i}\right)\right]+V\left(\mathbf{r}_{1}, \mathbf{r}_{2}\right),
$$

with $V_{\text {eff }}\left(\mathbf{r}_{i}\right)$ representing the effective potential for the $i$ th electron, and $V\left(\mathbf{r}_{1}, \mathbf{r}_{2}\right)$ is a two-body interaction operator which consists of the static Coulomb interaction, $1 /\left|\mathbf{r}_{1}-\mathbf{r}_{2}\right|$, and the effective dielectronic interaction [23-25]. The timedependent interaction operator between the atom and laser pulse $D(t)$ is written, in the dipole approximation and velocity gauge as

$$
D(t)=-\mathbf{A}(t) \cdot\left(\mathbf{p}_{1}+\mathbf{p}_{2}\right),
$$

where $\mathbf{p}_{1}$ and $\mathbf{p}_{2}$ represent the momenta of the two-valence electrons, and the vector potential $\mathbf{A}(t)$ is given by

$$
\mathbf{A}(t)=\mathbf{A}_{0} f(t) \cos (\omega t)
$$

with $\mathbf{A}_{0}$ being the amplitude of the vector potential and $\omega$ the photon frequency. $f(t)$ is the temporal envelope function of the laser pulse. Unless otherwise noted, $f(t)$ is chosen to have a cosine-squared shape, $f(t)=\cos ^{2}(\pi t / 2 \tau)$, where $\tau$ represents the full width at half maximum (FWHM) of the vector potential for the laser field. The integration time for the cosine-squared pulse is taken from $-\tau$ to $\tau$.

In order to solve Eq. (1) we expand the time-dependent wave function $\Psi\left(\mathbf{r}_{1}, \mathbf{r}_{2} ; t\right)$ on the atomic basis [19-22]:

$$
\Psi\left(\mathbf{r}_{1}, \mathbf{r}_{2} ; t\right)=\sum_{n, L, M} C_{E_{n} L M}(t) \Psi\left(\mathbf{r}_{1}, \mathbf{r}_{2} ; E_{n}\right) .
$$

Note that the above expansion of the total time-dependent wave function in terms of the atomic basis states is essentially equivalent to the time-dependent numerical wave function which is propagated by the finite-difference method, provided that the atomic basis states we employ form a (quasi-)complete set. Now, by substituting Eq. (5) into Eq. (1), we obtain a set of first-order differential equations for the time-dependent coefficients, $C_{E_{n} L M}(t)$, for the $n$th atomic state with a total orbital quantum number $L$, its projection $M$, and the eigenenergy $E_{n}$, which reads

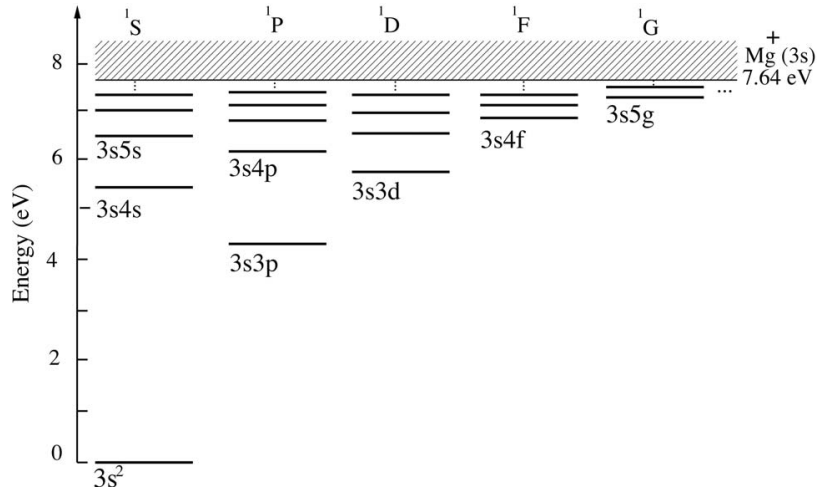

FIG. 1. Relevant energy levels of Mg.

$$
\begin{aligned}
& i \frac{d}{d t} C_{E_{n} L M}(t)=\sum_{n^{\prime}, L^{\prime}, M^{\prime}}\left[E_{n} \delta_{n n^{\prime}} \delta_{L L^{\prime}} \delta_{M M^{\prime}}\right. \\
& \text { - } \left.D_{E_{n} L M, E_{n^{\prime} L^{\prime} M^{\prime}}}(t)\right] C_{E_{n^{\prime} L^{\prime} M^{\prime}}}(t) .
\end{aligned}
$$

Note that Eq. (6) is valid for both LP and CP laser pulses. The quantities $D_{E_{n} L M, E_{n^{\prime} L^{\prime} M^{\prime}}}(t)$ represent the dipole matrix elements calculated between the states defined by the quantum numbers $\left(E_{n}, L, M\right)$ and $\left(E_{n^{\prime}}, L^{\prime}, M^{\prime}\right)$. The above set of differential equations for the time-dependent coefficients, $C_{E_{n}, L, M}(t)$, is solved, assuming that the $\mathrm{Mg}$ atom is initially in its ground state, $3 s^{21} S$, i.e.,

$$
\left|C_{E_{n} L M}(t=-\tau)\right|^{2}=\delta_{n 3} \delta_{L 0} \delta_{M 0} .
$$

Since we start from the singlet ground state and the singlettriplet transitions for the $\mathrm{Mg}$ atom are extremely weak, we can safely neglect the contribution of all triplet states. The relevant energy levels are presented in Fig. 1. As we have already implied in Eq. (6), owing to the dipole selection rules for laser polarization, namely, $\Delta M=+1$ for right $\mathrm{CP}$ light and $\Delta M=0$ for LP light, the allowed transitions from the ground state by the single-photon absorption with $\mathrm{CP}$ light are only between states $|L, M=L\rangle \rightarrow\left|L^{\prime}=L+1, M^{\prime}=L+1\right\rangle$ in comparison to the allowed transitions with LP light, $|L, M=0\rangle \rightarrow\left|L^{\prime}=L+1, M^{\prime}=0\right\rangle$. Figures 2(a) and 2(b) illustrate the multiphoton ionization paths according to the dipole selection rules for LP and CP light, respectively. Once the solution to Eq. (6) is obtained, the ionization yield $Y$ and the photoelectron yield $d P / d E$ at the end of the pulse can be calculated from the following relations:

$$
\begin{gathered}
Y=1-\sum_{n, L, M,\left(E_{n}<0\right)}\left|C_{E_{n} L M}(t=+\tau)\right|^{2}, \\
\left.\frac{d P}{d E}\right|_{E=E_{e}}=\sum_{L, M,\left(E_{n}=E_{e}\right)}\left|C_{E_{n} L M}(t=+\tau)\right|^{2},
\end{gathered}
$$

where $E_{e}$ represents the photoelectron energy of interest.

\section{IONIZATION BY THE SECOND HARMONIC OF A Ti:SAPPHIRE LASER}

In this section, we will present representative numerical results and discussion for multiphoton ionization by the fs 
(a)

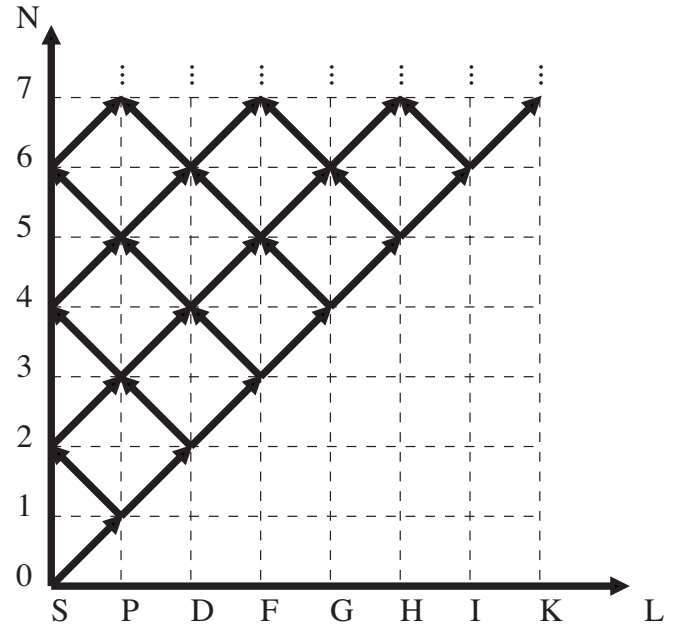

(b)

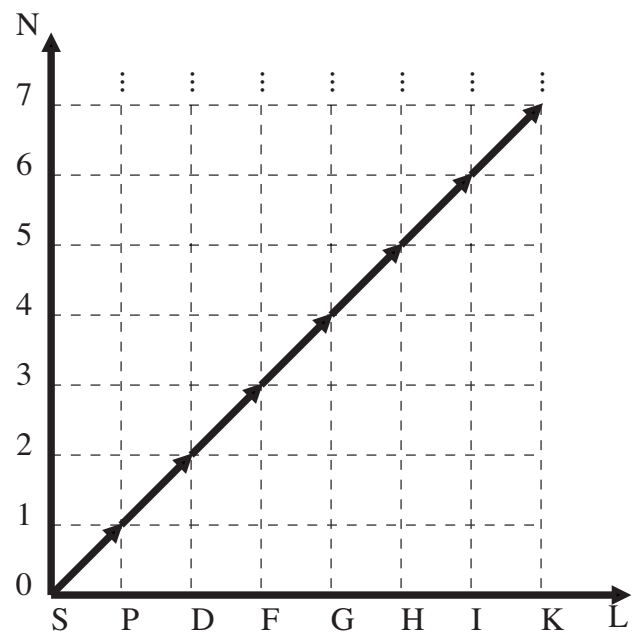

FIG. 2. (a) The allowed ionization paths in multiphoton ionization from an $S(L=0)$ initial state using (a) linearly polarized light and (b) right circularly polarized light.

pulse with a photon energy of $3 \mathrm{eV}$, which corresponds to the second harmonic of a Ti:sapphire laser. Since the ionization potential of $\mathrm{Mg}$ is $7.64 \mathrm{eV}$, at least three photons are needed for ionization. The intensity range we have considered for the numerical calculations is from $10^{11} \mathrm{~W} / \mathrm{cm}^{2}$ up to $10^{14} \mathrm{~W} / \mathrm{cm}^{2}$. At $10^{14} \mathrm{~W} / \mathrm{cm}^{2}$ the Keldysh parameter is 1.5 , suggesting that the transition of the ionization mechanism from multiphoton ionization to tunneling ionization takes place. The atomic basis states we need to solve the TDSE are constructed in a box size of $600 \mathrm{a}$.u. for the total angular momenta up to $L=9$ with 1000 states for each angular momentum. To check the numerical convergence we have increased the box size up to $1000 \mathrm{a} . \mathrm{u}$. together with the increased number of total orbital angular momenta up to $L$ $=15$ for each given intensity. It turned out that the basis states constructed in a box of $600 \mathrm{a} . \mathrm{u}$. for the total angular momenta up to $L=9$ with 1000 states in each angular momentum are sufficient to obtain the reasonable convergence in terms of the total ionization yield as well as the PES. As for the laser parameters, the temporal envelope function has been assumed to be cosine-squared with a duration of $50 \mathrm{fs}$ (FWHM) unless otherwise noted.
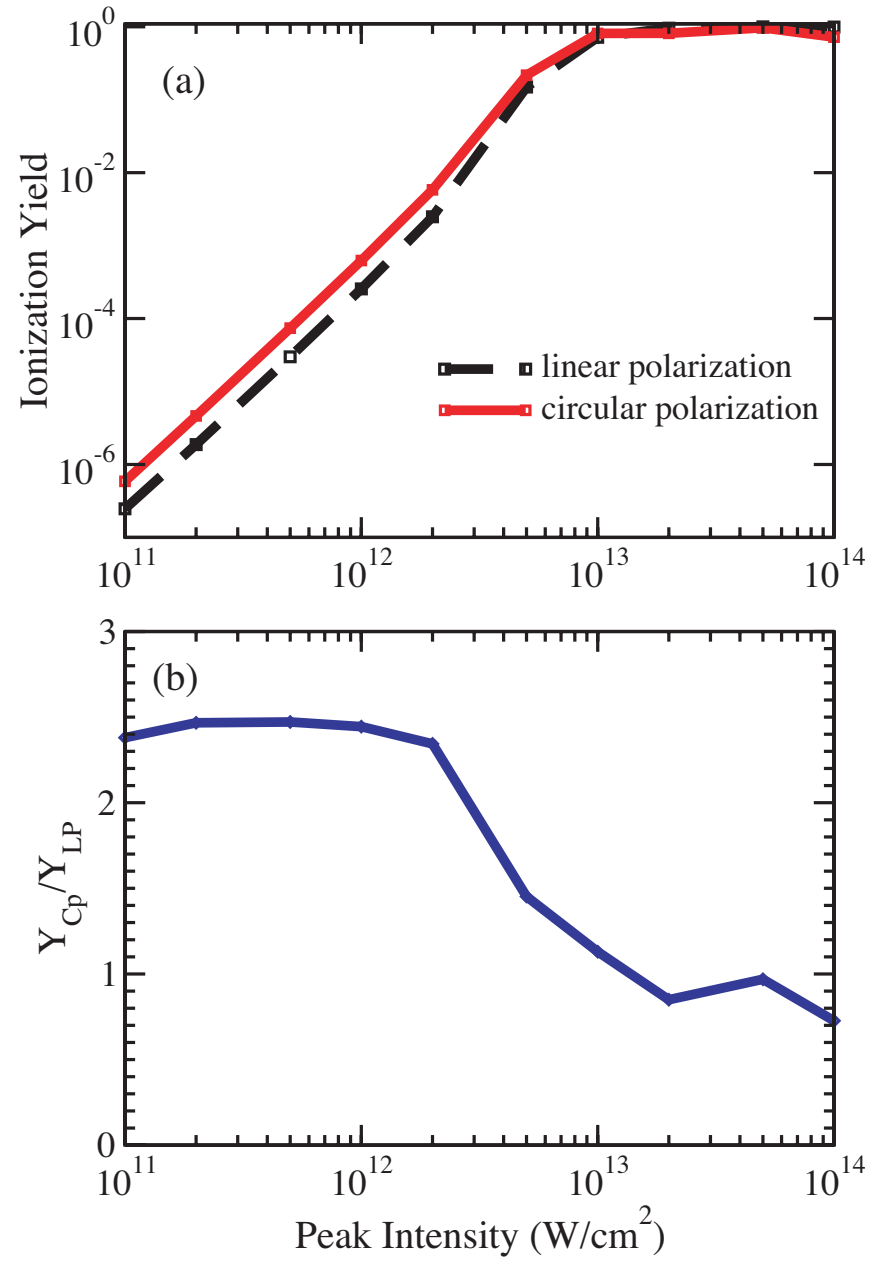

FIG. 3. (Color online) (a) Ionization yield as a function of peak intensity for the linearly (dashed) and circularly polarized (solid) laser pulses at the photon energy of $3 \mathrm{eV}$. (b) Ratio of the ionization yield by the circularly polarized pulse, $Y_{C P}$, to that by the linearly polarized pulse $Y_{L P}$. The pulse duration is $50 \mathrm{fs}$ (FWHM).

\section{A. Ionization yield}

The ionization yield is shown in Fig. 3(a) as a function of peak intensity for the CP (solid) and LP (dashed) pulses. For the pulse duration of $50 \mathrm{fs}$ (FWHM) we have chosen, both curves appear as almost straight lines, up to the peak intensity of $3 \times 10^{12} \mathrm{~W} / \mathrm{cm}^{2}$, with a slope of $\sim 3.07$, indicating that our results agree well with the prediction of lowest-order perturbation theory (LOPT). Namely, at least three photons are needed to ionize the $\mathrm{Mg}$ atom: Recall that the ionization yield is proportional to $I^{N}$ for $N$-photon ionization in LOPT, resulting in the slope of $N$ if the ionization yield is plotted as a function of peak intensity, $I$, in the log-log scale. For peak intensities higher than $6 \times 10^{12} \mathrm{~W} / \mathrm{cm}^{2}$, saturation starts to take place. Figure 3(a) also suggests that ionization by the $\mathrm{CP}$ laser pulse is more efficient than the LP pulse. Figure 3(b) presents the ratio between the ionization yield by the $\mathrm{CP}$ and LP pulses, $Y_{C P} / Y_{L P}$, as a function of peak intensity. For peak intensities up to $2 \times 10^{12} \mathrm{~W} / \mathrm{cm}^{2}$, the ionization yield by the CP pulse is about 2.44 times larger than that by the LP pulse, which compares very well with the ratio of the three- 


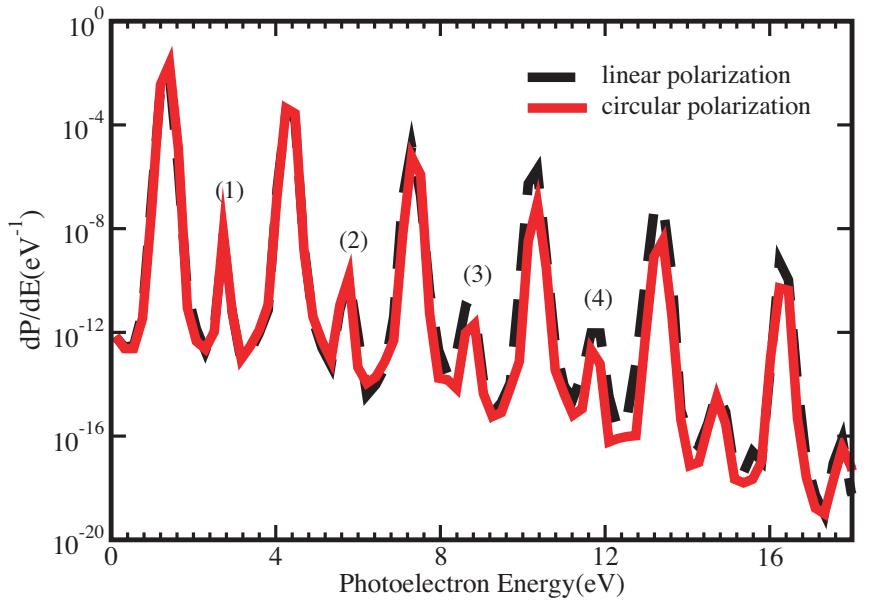

FIG. 4. (Color online) Photoelectron energy spectra by the linearly (dashed) and circularly polarized (solid) laser pulses at the photon energy of $3 \mathrm{eV}$. The pulse duration is $50 \mathrm{fs}$ (FWHM) and the peak intensity is $2 \times 10^{12} \mathrm{~W} / \mathrm{cm}^{2}$.

photon ionization cross sections at this photon energy, $\sigma_{C P}^{(3)} / \sigma_{L P}^{(3)}=2.1$ [26], suggesting that the ionization processes under our conditions are essentially well-described by the perturbation theory as long as the total ionization yield is concerned. It is interesting to point out that this ratio for $\mathrm{Mg}$ compares very well with the ratio 2.5 reported for the singlevalence-electron atoms $[14,17]$. This agreement is perhaps due to the fact that, starting from the ground state with the same $S$ symmetry, it is rather a geometric factor (i.e., angular coefficient) of the involved dipole matrix elements with increasing total orbital angular momenta, that plays an important role for the few-photon ionization process, at least under low intensities. This qualitatively explains why ionization by the CP field is more efficient by the LP field. Our timeindependent calculations for the multiphoton ionization cross sections of $\mathrm{Mg}$ [26] show that, when more than four photons are needed for ionization, ionization by the LP field starts to become more efficient for a wide range of photon energy, which can be qualitatively understood that photoionization by the LP field has more chance to be close to resonance with bound states than the $\mathrm{CP}$ field, and also there are accessible continua for the LP field.

\section{B. Photoelectron energy spectra}

In Fig. 4 we present representative PES by the CP (solid) and LP (dashed) pulses at the peak intensity of 2 $\times 10^{12} \mathrm{~W} / \mathrm{cm}^{2}$. As it goes to the higher order of ATI, the height of the ATI peaks by the LP pulse is more than one order of magnitude larger than that by the CP pulse. It is interesting to note that, in addition to the main ATI peaks, there appear subpeaks between them for both CP and LP pulses, which is somehow reminiscent of the results in the literature [27] in which near-resonant as well as off-resonant two-photon ionization of $\mathrm{H}$ has been numerically studied. In Fig. 4, the height of the subpeaks is about 5-6 orders of magnitude smaller than that of the main peaks. Related to our findings, we note that multiphoton ionization and ATI in
Mg using the second harmonic of a Ti:sapphire laser with LP was theoretically [28] as well as experimentally [29] studied. In the former paper the origin of the subpeaks is attributed to the combination of the following two processes: (a) a twophoton coupling of the $\mathrm{Mg}$ ground state, $3 s^{21} \mathrm{~S}$, to the $3 s 5 s^{1} S$ state and (b) a three-photon coupling of the ground state to the $3 p 4 s^{1} P$ autoionizing state, while in the latter paper the authors speculated that the subpeaks originate from the two different processes, (a) four-photon ionization of $\mathrm{Mg}$ leaving the parent ion in the excited ionic state, $\operatorname{Mg}^{+}(3 p)$, and (b) three-photon ionization of $\mathrm{Mg}$ followed by five-photon ionization of $\mathrm{Mg}^{+}(3 s)$, resulting in double ionization to produce $\mathrm{Mg}^{2+}$. The authors of Ref. [29], however, concluded that the physical origin of the subpeaks of $\mathrm{Mg}$ is not very clear and left it as an open question for further investigation.

\section{Origin of the subpeaks in the photoelectron energy spectra}

How do we understand the physical origin of the subpeaks in the PES? If the subpeaks arise from some photoionization processes involving four or five photons to leave the ionic core in some excited state, the height of the subpeaks with respect to the main ones would be even much smaller than they appear in Fig. 4 at the peak intensity of 2 $\times 10^{12} \mathrm{~W} / \mathrm{cm}^{2}$, assuming the typical excitation or ionization efficiency involving four or five photons. The subpeaks cannot be attributed to some intensity dependent effects, either: The ponderomotive shift is as small as $0.032 \mathrm{eV}$ at 2 $\times 10^{12} \mathrm{~W} / \mathrm{cm}^{2}$, and no states will come into resonance during the pulse.

The first important clue comes from the observation in Fig. 4 that the subpeaks appear for both LP and CP pulses with an equidistant energy separation corresponding the photon energy. This implies that the subpeaks are essentially ATI signals originating from some bound state(s) accessible for both LP and CP pulses. Thus, we speculate that the subpeaks are somehow related to the bound states with $P$ and/or $D$ symmetries excited by the single- and two-photon absorption, respectively: Recall that ionization requires at least three-photon absorption and therefore there are two intermediate states. Furthermore, from the energy consideration about the location of the subpeaks in the PES, the primary suspect is $3 s 3 p$ located at $4.35 \mathrm{eV}$ from the ground state. From the viewpoint of laser detuning, on the other hand, this state seems too far to be excited by the laser pulse with $3 \mathrm{eV}$ photon energy. The single-photon detuning is as much as $1.3 \mathrm{eV}$. Whether this state can be the origin of the subpeaks needs to be theoretically tested.

To verify our interpretation, we have repeatedly solved the TDSE after removing one of the bound states under suspicion, and compared the obtained results to see if there is any change in the appearance of the subpeaks. In Fig. 5, we present representative results obtained by removing the bound $3 s 3 p$ (dashed) and $3 s 5 s$ (dot-dashed) states, respectively, in comparison to the original results (solid) with all atomic states included during the time-propagation. Clearly, the removal of the $3 s 5 s$ state does not change the main feature of the PES as expected, as shown by the dot-dashed line 


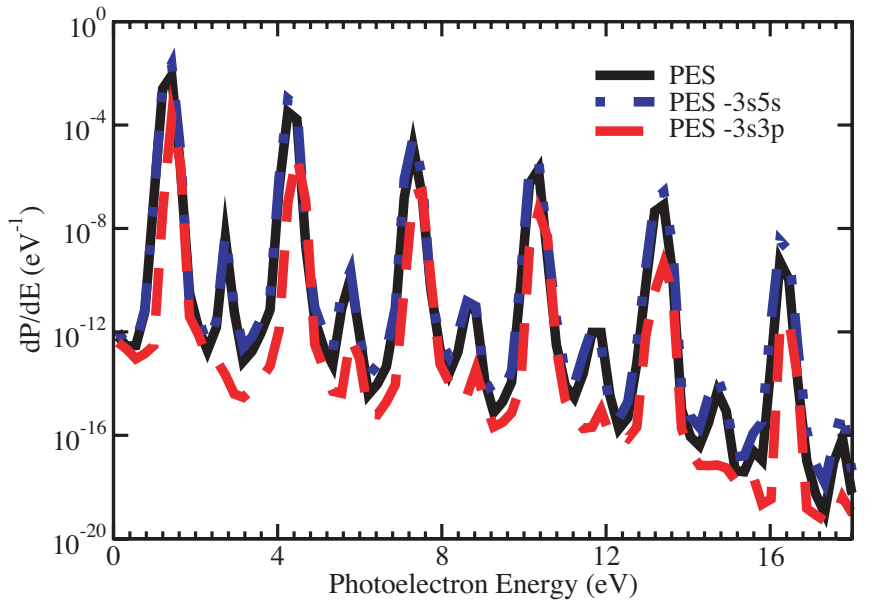

FIG. 5. (Color online) Comparison of the photoelectron energy spectra by the linearly polarized pulse at the photon energy of $3 \mathrm{eV}$ when the $3 s 3 p$ (dashed) and $3 s 5 s$ (dot-dashed) bound states of $\mathrm{Mg}$ are removed from the atomic basis when solving the timedependent Schrödinger equation. The pulse duration and peak intensity are the same as in Fig. 4.

in Fig. 5: the subpeaks still appear without any change. When the $3 s 3 p$ state is removed during the time propagation, however, we see the reduction in the height of the main and subpeaks in the PES. The reason for the reduction in the height of the main peaks can be easily found as follows. The main peaks reduce their heights by removing the $3 s 3 p$ state since the strong $3 s^{2}-3 s 3 p$ transition has a very large contribution to multiphoton ionization from the ground state. In other words, this reduction is due to the significant but nonresonant contribution of the $3 s 3 p$ state in multiphoton ionization: Recall that the main peaks appear as a result of the nonresonant ionization directly from the ground state. Regarding the subpeaks the one located at $2.8 \mathrm{eV}$ in Fig. 5 almost disappears upon the removal of the $3 s 3 p$ state, while the subpeaks at higher energy do not really disappear but reduce their heights by two to three orders of magnitude. These results suggest that the subpeaks are essentially due to multiphoton ionization from the $3 s 3 p$ state which is located at $4.35 \mathrm{eV}$ from the ground state and off-resonant as much as $1.3 \mathrm{eV}$ for the photon energy of $3 \mathrm{eV}$. In other words, regardless of the large detuning, the off-resonant but real excitation of the $3 s 3 p$ state takes place by some excitation mechanisms which we need to identify.

We can think of two possible excitation mechanisms of the far off-resonant $3 s 3 p$ state. The first possibility is that the off-resonant excitation might take place by the spectral wing of the pulse. The second possibility is that the off-resonant excitation might take place by the off-resonant Rabi coupling between the ground state and $3 s 3 p$. Our answer to the question of which is actually the case is postponed to Sec. III D. What is to be understood, for a moment, is that a far offresonant state can be somehow excited during the pulse. The smaller but remaining subpeaks at the higher energy of 5.8, $8.8 \mathrm{eV}$, and so on upon the removal of $3 s 3 p$ shown by the dashed line in Fig. 5 can be understood that there still remains a small and similar contribution of other bound states which can be off-resonantly excited. Note that the use of the

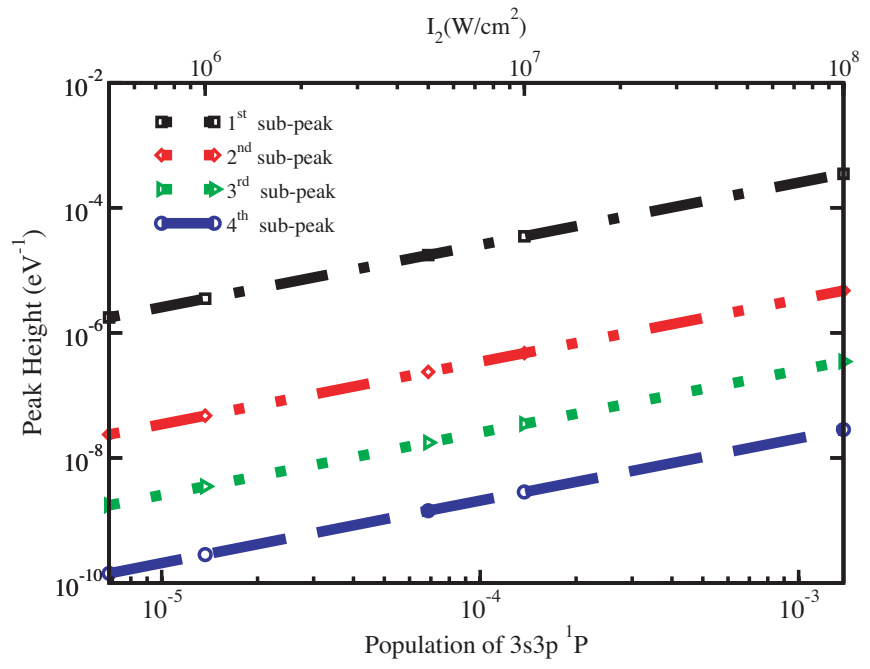

FIG. 6. (Color online) The height of the first four subpeaks [labeled as (1)-(4) in Fig. 4] subject to the two-color (main and auxiliary) laser pulses as a function of population of the $3 s 3 p$ state (labeled on the bottom horizontal axis) and accordingly peak intensity of the auxiliary laser (labeled on the top horizontal axis). The peak intensity and the photon energy of the main laser are $10^{12} \mathrm{~W} / \mathrm{cm}^{2}$ and $3 \mathrm{eV}$, respectively. The photon energy of the auxiliary laser is $4.35 \mathrm{eV}$. Both lasers are linearly polarized with the same pulse durations of $50 \mathrm{fs}$ (FWHM).

atomic states during the time propagation enabled us to carry out the above numerical comparison. If we had integrated the wave function itself by other methods, this kind of numerical comparison would not be easily done.

As a second test, we have also carried out the timedependent calculations with a very weak auxiliary laser pulse with the photon energy of $4.35 \mathrm{eV}$ and the duration of $50 \mathrm{fs}$ (FWHM). The peak intensity has been chosen to be in the range $\left(5 \times 10^{5}\right)-10^{8} \mathrm{~W} / \mathrm{cm}^{2}$, since the purpose of the auxiliary laser pulse is just to resonantly excite $3 s 3 p$ while maintaining a negligible contribution to the total photoelectron yield, since ionization from $3 s 3 p$ requires two photons by either auxiliary $(4.35 \mathrm{eV})$ or main $(3 \mathrm{eV})$ laser pulses. As for the main laser, the peak intensity has been kept constant at $10^{12} \mathrm{~W} / \mathrm{cm}^{2}$. In Fig. 6 we plot the height of the first four subpeaks as a function of population of the $3 s 3 p^{1} P_{1}$ state, which is naturally proportional to the peak intensity of the auxiliary laser as labeled on the horizontal axis at the top of Fig. 6. The slope of all these four plots is equal to 1, implying that there is a linear dependence of the height of the subpeaks on the population of the $3 s 3 p$ state. This is another confirmation that the origin of all the subpeaks are essentially off-resonant excitation of the $3 s 3 p$ state, from which multiphoton ionization takes place.

To our knowledge, the appearance of the subpeaks in the PES by experimentally available fs laser pulses has not yet been reported for other atoms such as $\mathrm{H}, \mathrm{He}$, and other rare gases. According to our interpretation described above, there is no wonder for that, since the appearance of the subpeaks requires a single-photon real excitation of an off-resonant bound state. If the photon energy employed for the theoretical calculations or experiments is in the range of one up to a 
few $\mathrm{eV}$, even the first excited states cannot be easily reached since they are located as high as $8.4 \mathrm{eV}$ (for $\mathrm{Xe}$ ) to $20.6 \mathrm{eV}$ (for $\mathrm{He}$ ) from the ground state. $\mathrm{Mg}$ is very different from the above-mentioned atoms in that the first excited states is located as low as $4.35 \mathrm{eV}$ from the ground state.

\section{Effects of the temporal profile of the laser pulse}

In Sec. III C we have carried out a comparative study to clarify the origin of the subpeaks in the PES, and identified that the off-resonant but real excitation of the $3 s 3 p$ state is the main mechanism. After pointing out the two possible mechanisms of the off-resonant excitation, the question of which is actually the case that has been left as an open question with a promise to be clarified in this subsection.

To clarify the off-resonant excitation mechanism, it is useful to examine the effects of the temporal profile of the laser pulses: Laser pulses with the same pulse duration (FWHM) but with different temporal profiles can lead to the different spectral profiles at the wing. Therefore, if the off-resonant excitation is induced by the spectral wing of the pulse, the height of the subpeaks would be very sensitive to the precise spectral profile of the laser pulse. Alternatively, if the offresonant excitation is due to the off-resonant Rabi coupling between the ground and $3 s 3 p$ state, the off-resonant excitation would mainly take place around the peak intensity where the Rabi coupling is maximum, and accordingly the height of the subpeaks must be rather insensitive to the precise temporal profile, provided that the pulse duration (in FWHM) and the peak intensity are maintained to be the same. Specifically we consider three different types of the temporal envelope function for the vector potential of the laser pulse defined in Eq. (4): (1) cosine-squared $f(t)$ $=\cos ^{2}(\pi t / 2 \tau) ;(2)$ hyperbolic secant $f(t)=\operatorname{sech}[2 \ln (2+\sqrt{3})$ $\times(t / \tau)$; (3) Gaussian $f(t)=\exp \left[-4 \ln 2(t / \tau)^{2}\right]$.

The numerical factors in each temporal envelope function, $f(t)$, are chosen so that $\tau$ represents the temporal width for the FWHM of the laser pulses. To obtain the numerical convergence, the integration time is taken from $-\tau$ to $\tau$ for the cosine-squared pulse, from $-5 \tau$ to $5 \tau$ for the hyperbolic secant pulse, and from $-3 \tau$ to $3 \tau$ for the Gaussian pulse, respectively. As one can easily verify by taking the Fourier transform, the spectral profile of the cosine-squared pulse has more broadened wings than the hyperbolic secant pulse, which has more broadened wings than the Gaussian pulse. The central part of the spectral profile, however, is almost the same for those three pulses. This implies that, if the offresonant excitation takes place by the spectral wing of the pulse, the use of the cosine-squared pulse would be most efficient to excite the off-resonant $3 s 3 p$ state, resulting in the most prominent subpeaks while the height of the main peaks remains almost the same for all three different temporal envelope functions.

In Fig. 7 we present the comparison of the PES for the pulses with cosine-squared (solid), hyperbolic secant (dashed), and Gaussian (dot-dashed) temporal profiles. Except for the temporal envelope function $f(t)$ and the pulse duration which is chosen to be $50 \mathrm{fs}$ (FWHM), all other laser

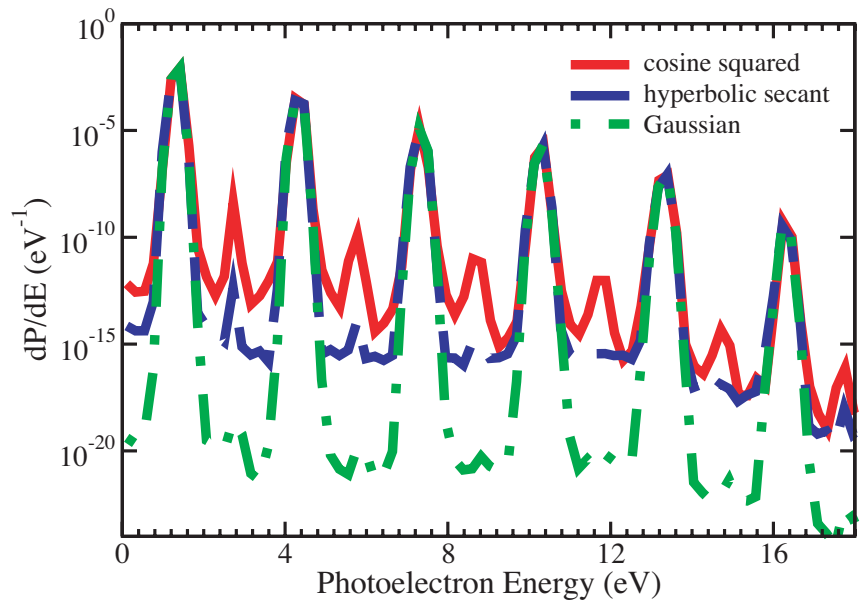

FIG. 7. (Color online) Comparison of the photoelectron energy spectra for the pulses with cosine-squared (solid), hyperbolic secant (dashed), and Gaussian (dot-dashed) temporal envelope functions having the same pulse duration of $50 \mathrm{fs}$ (FWHM). Polarization is linear for all cases and the photon energy and peak intensity are the same as in Fig. 4.

parameters are taken to be the same as those for Fig. 4. Figure 7 reveals the features we have mentioned above: The height of the first subpeak for the hyperbolic secant pulse is about four orders of magnitude smaller than the cosinesquared pulse. For the Gaussian pulse which has the narrowest spectral wings among the three pulses, we observe the further-reduced subpeaks with respect to the main peaks. Based on Fig. 7, we can conclude with confidence that the off-resonant excitation of $3 s 3 p$ is not induced by the offresonant Rabi coupling, but rather induced by the spectral wing of the pulse. Note that our results shown in Fig. 7 are for the transform-limited pulses with three different temporal envelope functions. From the experimental point of view, fs pulses with a 50-100 fs duration are not necessarily transform-limited, and they may have an extra bandwidth arising from some instability in the laser operation. Therefore, the comparison between theory and experiment have to be conducted with a great care.

\section{E. Effects of the laser pulse duration}

Due to the rapid advances for the laser technology, shorter and shorter laser pulses are now becoming available in laboratories. Even in the intensity range where multiphoton ionization rather than tunneling ionization is the main mechanism for ionization which is our case, we can expect that the PES would reveal a strong dependence on the pulse duration, since the spectral profile becomes broader for shorter pulses. In Figs. 8(a)-8(c) we show the PES for the LP pulse with a cosine-squared temporal envelope function for three different pulse durations, $\tau=50,20$, and $10 \mathrm{fs}$ (FWHM), with the same peak intensity of $2 \times 10^{12} \mathrm{~W} / \mathrm{cm}^{2}$. As the pulse duration decreases the peaks broaden with some decrease in their magnitudes due to the shorter pulse durations. Moreover, the relative height of the subpeaks with respect to the main peaks in the PES increases for the shorter pulse durations. 

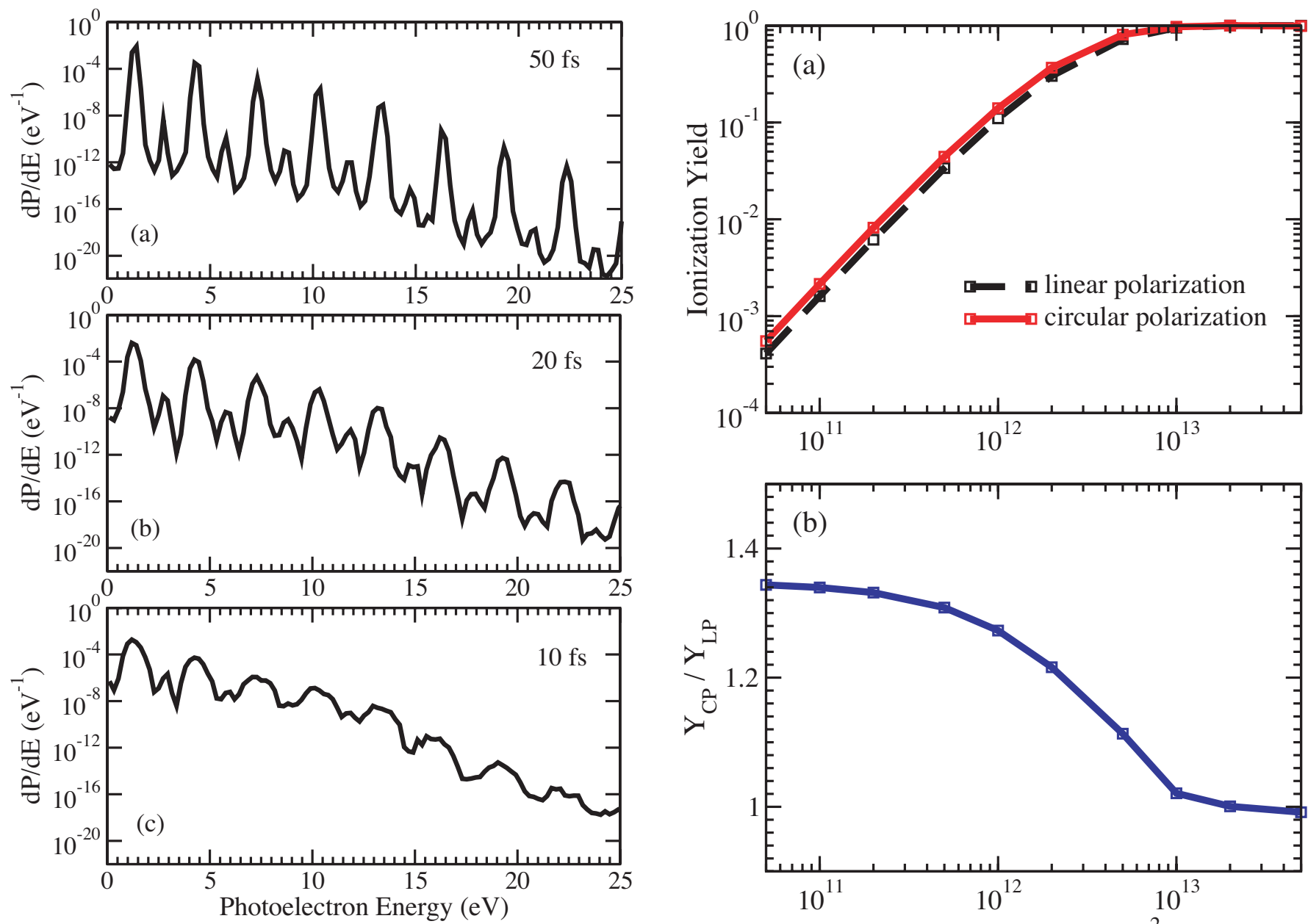

FIG. 8. Photoelectron energy spectra at the photon energy of $3 \mathrm{eV}$ and the peak intensity of $2 \times 10^{12} \mathrm{~W} / \mathrm{cm}^{2}$ for three different pulse durations, (a) 50, (b) 20, and (c) 10 fs (FWHM). Laser polarization is linear.

This can be simply interpreted that the excitation of the offresonant $3 s 3 p$ state, which we have identified as the origin of the subpeaks by the spectral wing of the pulse, becomes more efficient for shorter pulses with respect to the main peaks. Note that the main peaks originate from the nonresonant multiphoton ionization directly from the ground state, while the subpeaks originate from the nonresonant multiphoton ionization from the excited states such as $3 s 3 p$, etc. Obviously the height of the subpeaks is more sensitive to the pulse duration or equivalently the spectral profile of the pulse compared with the main peaks.

\section{IONIZATION BY THE THIRD HARMONIC OF A Ti:SAPPHIRE LASER}

In Sec. III, we have presented results and discussion for ionization induced by the second harmonic of a Ti:sapphire laser which requires three photons to reach the ionization threshold. That is, there are two virtual intermediate states below the threshold. Given the Ti:sapphire-based fs pulses, another photon energy that can be fairly easily obtained is the third harmonic of the Ti:sapphire laser. More specifically,

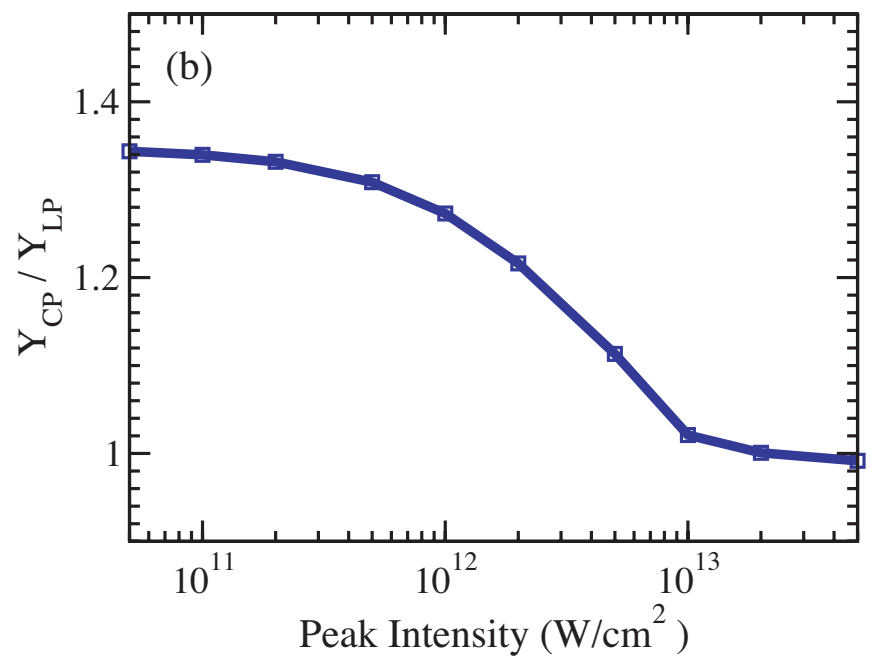

FIG. 9. (Color online) (a) Ionization yield as a function of peak intensity for the linearly (dashed) and circularly polarized (solid) laser pulses at the photon energy of $4.65 \mathrm{eV}$. (b) Ratio of the ionization yield by the circularly polarized pulse, $Y_{C P}$, to that by the linearly polarized pulse $Y_{L P}$. The pulse duration is $50 \mathrm{fs}$ (FWHM).

we assume that the photon energy of the third harmonic is $4.65 \mathrm{eV}$. Two photons are needed to reach the ionization threshold and therefore there is only one virtual intermediate state below the threshold. This means that, compared to the second harmonic, the use of the third harmonic is even more convenient for the clear understanding of the calculated results.

First, we present the ionization yield in Fig. 9(a) as a function of the peak intensity for the CP (solid) and LP (dashed) pulses. The pulse duration is 50 fs (FWHM). For both CP and LP pulses, the ionization yields increase linearly with the peak intensity up to $I=3 \times 10^{12} \mathrm{~W} / \mathrm{cm}^{2}$ where saturation starts to take place. The slope of these curves is $\sim 1.85$ in the intensity range up to $I=2 \times 10^{12} \mathrm{~W} / \mathrm{cm}^{2}$, which is slightly smaller than the prediction by LOPT, which is 2 . Since the first excited state $3 s 3 p$ is located at $4.35 \mathrm{eV}$, this state is now near-resonant since the photon energy is $4.65 \mathrm{eV}$. In Fig. 9(b) we present the ratio between the ionization yield by the CP and LP pulses, $Y_{C P} / Y_{L P}$, as a function of peak intensity. At low laser intensities the ratio is about 


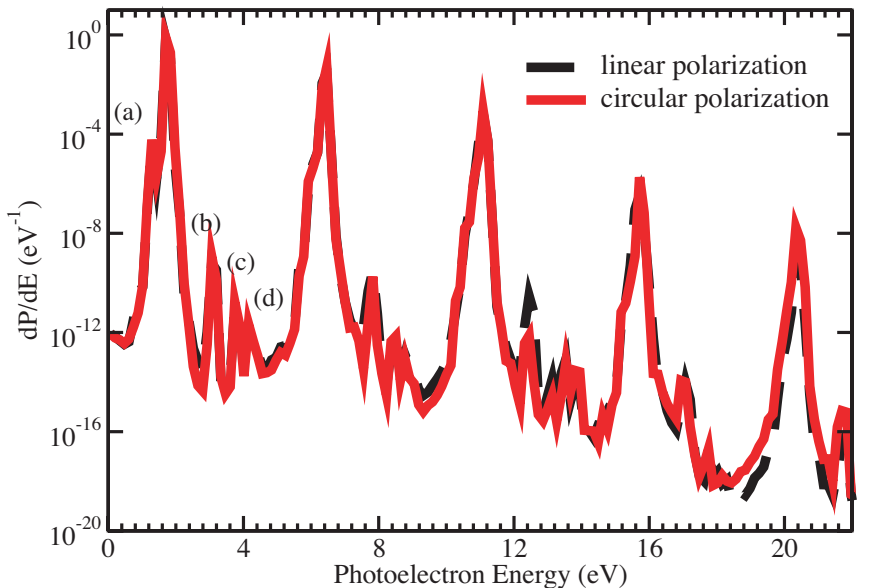

FIG. 10. (Color online) Photoelectron energy spectra by the linearly (dashed) and circularly polarized (solid) laser pulses at the photon energy of $4.65 \mathrm{eV}$. The pulse duration and peak intensity are $50 \mathrm{fs}(\mathrm{FWHM})$ and $10^{12} \mathrm{~W} / \mathrm{cm}^{2}$, respectively.

1.35 and this is rather close to the ratio of two-photon ionization cross sections $\sigma_{C P}^{(2)} / \sigma_{L P}^{(2)}=1.42$ [26], which is very similar to the value of the ratio for the single-valenceelectron atoms $[14,17]$. The physical reason of why ionization by the CP field is more efficient than the LP is qualitatively similar to the case for the three-photon ionization we have already discussed in Sec. III A. At higher intensities this ratio becomes smaller since saturation for the $\mathrm{CP}$ pulse starts at slightly lower intensity than the LP pulse, as one can see in Fig. 9(a). As long as the peak intensity is well below the saturation intensity, ionization by the $\mathrm{CP}$ pulse is more efficient than the LP pulse, which is the same tendency we have seen for the second harmonic pulse in Fig. 3.

In Fig. 10 we plot the PES by the CP (solid) and LP pulses (dashed) with the pulse duration of 50 fs (FWHM) and the peak intensity of $10^{12} \mathrm{~W} / \mathrm{cm}^{2}$. The PES by the CP and LP laser pulses look very similar. Almost all of the subpeaks appear in the PES by both CP and LP pulses. Note that this observation resembles the previous result we have shown in Fig. 4 for the second harmonic pulse. The only difference between them is that there are more than one subpeak [labeled as (b)-(d) for the first group of the subpeaks in Fig. 10] appearing between the main peaks. Moreover there is a small "spike" [labeled as (a) in Fig. 10] on the left side of the first main peak. Similar subpeaks and also spikes (not clearly resolved in the second main peak and after) appear successively at the higher energy region in Fig. 10. It turned out that these substructures are regularly apart by the photon energy, $4.65 \mathrm{eV}$, which also suggests that perhaps all of these substructures originate from some bound state(s). Furthermore, the fact that almost all subpeaks appear for both CP and LP pulses implies that bound states which are accessible by both polarization through the single-photon absorption would be the origin of them. Thus, $3 \sin p(n=3,4,5, \ldots$, states are under suspect, again.

The procedure we have taken to identify the origin of these subpeaks in the PES is quite similar to that we have done for the second harmonic case. In Figs. 11(a)-11(d) we summarize our results calculated for the LP pulse at the peak
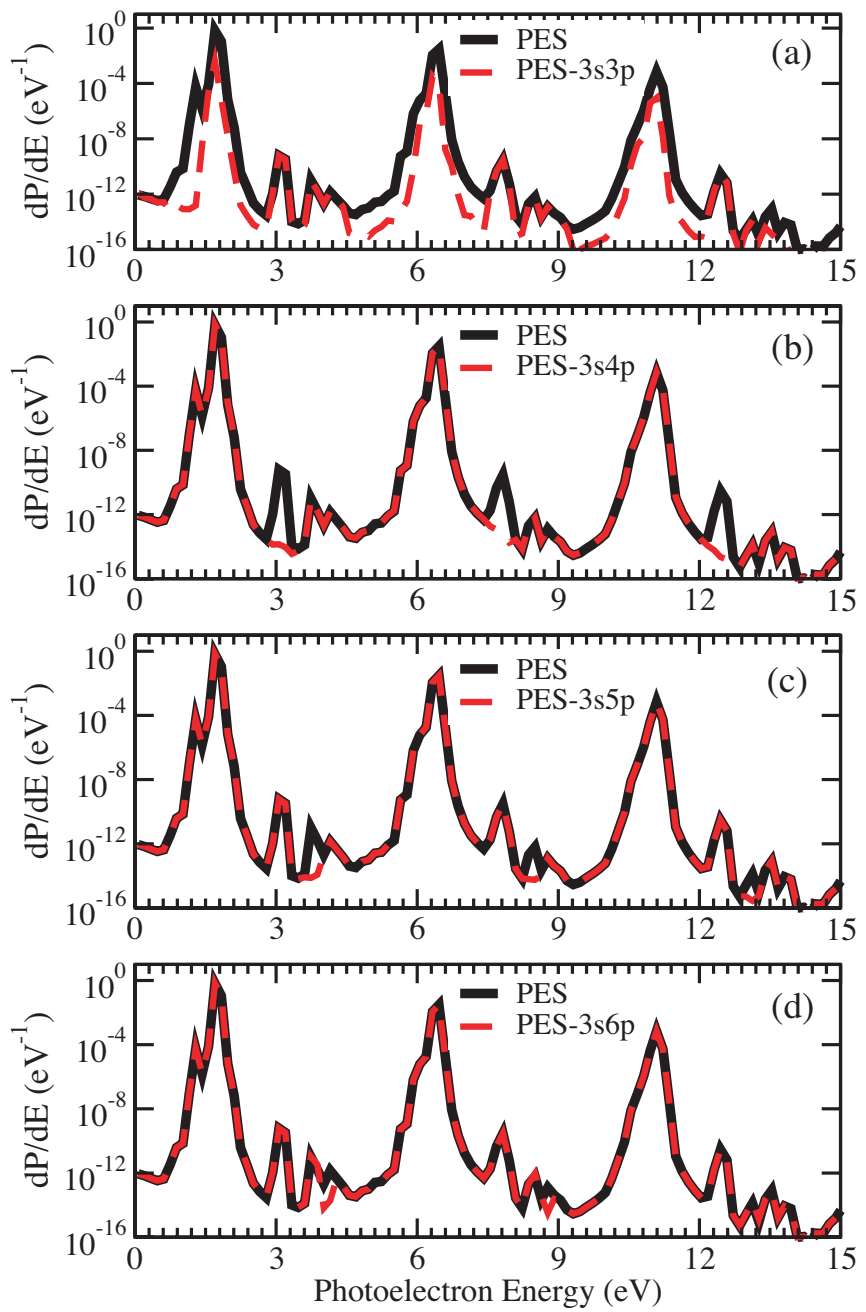

FIG. 11. (Color online) Comparison of the photoelectron energy spectra by the linearly polarized pulse at the photon energy of $4.65 \mathrm{eV}$ when the (a) $3 s 3 p$, (b) $3 s 4 p$, (c) $3 s 5 p$, and (d) $3 s 6 p$ bound states of $\mathrm{Mg}$ are removed from the atomic basis when solving the time-dependent Schrödinger equation. The pulse duration and peak intensity are the same as in Fig. 10.

intensity of $10^{12} \mathrm{~W} / \mathrm{cm}^{2}$, where the results obtained by removing a particular bound state, (a) $3 s 3 p$, (b) $3 s 4 p$, (c) $3 s 5 p$, and (d) $3 s 6 p$, upon solving the TDSE, are compared by the result with complete calculations including all atomic states. When the $3 s 3 p$ state is removed [Fig. 11(a)], the spike on the left side of the fist main peak disappears. Moreover, the height of the main peaks is drastically reduced since the $3 s 3 p$ state plays a very important contribution to the ionization process. It is clear that the spike [labeled as (a) in Fig. 10] is due to the excitation of the $3 s 3 p$ state. In this particular case the laser detuning is $0.3 \mathrm{eV}$ with respect to the $3 s 3 p$ state. That is, the small spike, located at $0.89 \mathrm{eV}$, corresponds to the single-photon ionization process from the $3 s 3 p$ excited state. Similarly, by removing the $3 \sin p(n=4,5,6)$ states different one of the subpeaks disappears, as can be seen in Figs. 11(b)-11(d). This indicates that the physical origin of the subpeaks in Fig. 10 for the third harmonic pulse is quite similar to that we have found for the second harmonic pulse: Essentially off-resonant bound states such as 
$3 \operatorname{snp}(n=4,5,6, \ldots$,$) are the origin of the subpeaks. We$ would like to note that those states are located at 6.12, 6.78, and $7.09 \mathrm{eV}$, respectively, and accordingly the detuning is $1.47,2.13$, and $2.44 \mathrm{eV}$. It is rather surprising that such far off-resonant bound states can contribute to the substructure in the PES.

\section{CONCLUSIONS}

In conclusion we have theoretically studied multiphoton ionization of $\mathrm{Mg}$ by linearly and circularly polarized fs laser pulses. The photon energies we have specifically chosen correspond to the second and third harmonics of a Ti:sapphire laser. For both photon energies the ionization yields have been found to be larger for the circularly polarized pulse. Since the $\mathrm{Mg}$ atom has a rather dense level structure, its fingerprints have been seen in the photoelectron energy spectra as an appearance of the subpeaks in addition to the ordinary main peaks. By the detailed comparative study we have clearly identified the origin of those subpeaks as ATI originating from some bound states which are far off-resonantly excited by the spectral wing of the pulse. As a result, the position and the magnitude of the subpeaks in the photoelectron energy spectra depend on the photon energy and the temporal profile of the pulse. We have also shown that the magnitude of the subpeaks critically depends on the spectral profile of the pulse, implying that the use of the different temporal envelope functions, even with the same pulse duration (FWHM), may result in a different height of the subpeaks. From the experimental point of view, this suggests that, if the pulse employed in experiments is not transformlimited, subpeaks may appear much more significant, since the extra spectral bandwidth can induce much more efficient excitation of the off-resonant states.

\section{ACKNOWLEDGMENTS}

G.B. acknowledges financial support from the Japan Society for the Promotion of Science (JSPS) and the hospitality at Kyoto University during her stay. The work by T. N. was supported by a Grant-in-Aid for scientific research from the Ministry of Education and Science of Japan.
[1] P. Agostini, F. Fabre, G. Mainfray, G. Petite, and N. K. Rahman, Phys. Rev. Lett. 42, 1127 (1979).

[2] L. F. DiMauro and P. Agostini, Adv. At., Mol., Opt. Phys. 35, 79 (1995).

[3] P. H. Bucksbaum, M. Bashkansky, R. R. Freeman, T. J. McIlrath, and L. F. DiMauro, Phys. Rev. Lett. 56, 2590 (1986).

[4] R. R. Freeman, P. H. Bucksbaum, H. Milchberg, S. Darack, D. Schumacher, and M. E. Geusic, Phys. Rev. Lett. 59, 1092 (1987).

[5] W. Nicklich, H. Kumpfmüller, H. Walther, X. Tang, H. Xu, and P. Lambropoulos, Phys. Rev. Lett. 69, 3455 (1992).

[6] P. B. Corkum, N. H. Burnett, and F. Brunel, Phys. Rev. Lett. 62, 1259 (1989).

[7] P. H. Bucksbaum, L. D. Van Woerkom, R. R. Freeman, and D. W. Schumacher, Phys. Rev. A 41, 4119 (1990).

[8] N. J. van Druten, R. Trainham, and H. G. Muller, Phys. Rev. A 51, R898 (1995).

[9] H. R. Reiss and V. P. Krainov, Phys. Rev. A 50, R910 (1990).

[10] A. Becker, L. Plaja, P. Moreno, M. Nurhuda, and F. H. M. Faisal, Phys. Rev. A 64, 023408 (2001).

[11] L. V. Keldysh, Sov. Phys. JETP 20, 1307 (1965).

[12] M. V. Ammosov, N. B. Delone, and V. P. Krainov, Sov. Phys. JETP 64, 1191 (1986).

[13] D. M. Volkov, Z. Phys. 94, 250 (1935).
[14] P. Lambropoulos, Phys. Rev. Lett. 28, 585 (1972).

[15] H. R. Reiss, Phys. Rev. Lett. 29, 1129 (1972).

[16] S. Klarsfeld and A. Maquet, Phys. Rev. Lett. 29, 79 (1972).

[17] Y. Gontier and M. Trahin, Phys. Rev. A 7, 2069 (1973).

[18] K. J. LaGattuta, Phys. Rev. A 43, 5157 (1991).

[19] X. Tang, H. Rudolph, and P. Lambropoulos, Phys. Rev. Lett. 65, 3269 (1990).

[20] X. Tang, H. Rudolph, and P. Lambropoulos, Phys. Rev. A 44, R6994 (1991).

[21] P. Lambropoulos, P. Maragakis, and J. Zhang, Phys. Rep. 305, 203 (1998).

[22] L. A. A. Nikolopoulos, G. Buica-Zloh, and P. Lambropoulos, Eur. Phys. J. D 26, 245 (2003).

[23] T. N. Chang, Many-body Theory of Atomic Structure and Photoionization (World Scientific, Singapore, 1993), p. 213.

[24] S. Mengali and R. Moccia, J. Phys. B 29, 1597 (1996).

[25] G. Buica-Zloh and L. A. A. Nikolopoulos, Eur. Phys. J. D 29, 201 (2004).

[26] G. Buica and T. Nakajima (unpublished).

[27] K. J. LaGattuta, Phys. Rev. A 47, 1560 (1993).

[28] Jian Zhang and P. Lambropoulos, Phys. Rev. Lett. 77, 2186 (1996).

[29] D. Xenakis, N. E. Karapanagioti, D. Charalambidis, H. Bachau, and E. Cormier, Phys. Rev. A 60, 3916 (1999). 